\title{
Lazos fuertes y proveedores múltiples de apoyo: comparación de dos formas de representación gráfica de las redes personales
}

\author{
Isidro MAYa Jariego y Daniel Holgado ${ }^{1}$ \\ Departamento de Psicología Social, Universidad de Sevilla \\ isidromj@us.es
}

Recepción: octubre 2005

Aceptación: noviembre 2005

\section{INTRODUCCIÓN}

La representación gráfica de las relaciones es un instrumento habitual del análisis de redes sociales. Linton Freeman (2004) la presenta como uno de los cuatro rasgos distintivos de dicho campo de estudio. Concretamente, (a) el análisis de redes sociales parte de la «intuición estructural» que atribuye un papel significativo a los patrones de relaciones entre actores sociales, (b) se basa en la recogida de datos empíricos sistemáticos, (c) utiliza modelos matemáticos o informáticos y (d) le da especial importancia a la representación visual.

Por ejemplo, es habitual que los investigadores traten de resumir y presentar sus resultados a través de la agrupación de individuos en cliques, la elaboración de sociogramas y árboles de parentesco, o la aplicación de diferentes formas de escalamiento multidimensional, entre otros procedimientos. Por lo general, estas estrategias sirven para identificar la existencia de agrupaciones de individuos o para mostrar conjuntos de actores que ocupan posiciones sociales equivalentes (Freeman, 2005). La representación visual de las relaciones tiene un enorme poder descriptivo y se cuenta entre los elementos del análisis de redes que suscita mayor interés e impacto entre los destinatarios de la investigación.

Las técnicas gráficas se utilizan preferentemente con fines exploratorios. Las imágenes permiten examinar los patrones de los datos relacionales, de modo que puedan ser contrastados con análisis estadísticos a continuación. Ese fue el en-

1 Esta investigación contó con la ayuda del Ayuntamiento de Alcalá de Guadára al estudio «Participación ciudadana y sentido de comunidad en Alcalá de Guadaíra: la implicación comunitaria de los universitarios alcalareños». Correspondencia con los autores: Isidro Maya Jariego, Departamento de Psicología Social, Universidad de Sevilla, Campus Ramón y Cajal, Calle Camilo José Cela s/n.41.018 Sevilla (Spain).E-mail: isidromj@us.es 
foque que seguimos para describir las diferentes formas de vida metropolitana en un grupo de jóvenes universitarios (Araya y Maya Jariego, 2005; Maya Jariego, Araya y Armitage, 2005). En primer lugar, clasificamos a los encuestados de acuerdo con el tiempo que pasaban en el lugar de residencia y en la ciudad donde cursaban los estudios. A continuación, representamos gráficamente las redes personales de diez universitarios que pasaban más del ochenta por ciento de su tiempo en el lugar de residencia, junto a las de otros diez que, por el contrario, sólo pasaban a lo sumo el veinte por ciento. A partir de esta comparación de casos, observamos diferencias en la composición y la estructura de las redes personales entre ambos grupos. Finalmente, realizamos una serie de análisis estadísticos con los que confirmar las expectativas que habíamos concebido con la representación visual.

Como vemos, las técnicas gráficas sirven para experimentar con los datos y generar hipótesis. Para una revisión amplia en ese sentido, puede consultarse el artículo de Freeman (2005), o la pormenorizada presentación de estrategias exploratorias de análisis con el programa Pajek (De Nooy, Mrvar y Batagelj, 2005). Sin embargo, la representación visual también puede utilizarse con otros fines:

- Sin ir más lejos, puede servir simplemente para comunicar las propiedades estructurales de las redes sociales. Las imágenes se han utilizado a lo largo de la historia en los estudios de redes sociales para describir y presentar los patrones de relación (Freeman, 2000).

- Por otro lado, puede aplicarse para obtener nueva información. El feedback gráfico genera reacciones y comportamientos susceptibles de análisis. Por ejemplo, la representación de las redes personales es útil para la entrevista cualitativa posterior de los encuestados (McCarty, Molina, Aguilar y Rota, 2004; Molina, McCarty, Aguilar y Rota, 2005). Sirve, entre otras cuestiones, para obtener información significativa con la que interpretar los datos relacionales.

- Finalmente, y entre otras posibilidades, proporciona información cualitativa que puede ser sometida a análisis y sistematización. Es decir, de algún modo, los gráficos son datos cualitativos con los que podemos llevar a cabo un análisis de contenido. Siguiendo ese procedimiento, en el caso de los universitarios ya mencionado, elaboramos una tipología de las redes personales agrupando los gráficos en función de tres dimensiones diferentes (Maya Jariego et al., 2005).

Sea cual sea el uso que demos a la visualización de las redes, resulta de interés conocer la percepción de los destinatarios -o, en su caso, usuarios- de la representación gráfica. La efectividad de la comunicación de las propiedades de una red social depende en cierto grado de cómo decodifica la información el receptor de la misma (McGrath y Blythe, 2004). Por eso es pertinente estudiar cómo perciben los individuos la representación de sus redes personales, o de las redes sociales de las que forman parte. 
Conocer de antemano la posible reacción de los receptores ayuda a perfilar la estrategia de representación gráfica. Pero no sólo es útil para diseñar la mejor forma de comunicar la información. Desde nuestro punto de vista, también puede (a) potenciar la fiabilidad de la investigación exploratoria, (b) optimizar la utilización del feedback gráfico en la entrevista cualitativa, y (c) contribuir a la pertinencia de intervenciones que utilizan el estímulo visual para promover el cambio personal o grupal ${ }^{2}$.

Con este estudio pretendemos conocer cómo perciben los individuos la representación gráfica de su red personal. En este tipo de visualización de redes ego-céntricas, que ha sido menos tratado que el de las redes completas, examinamos dos tipos de reacciones de los participantes. Primero, analizamos los comentarios de los encuestados cuando observan un gráfico que representa el espacio de primer orden en sus relaciones interpersonales (es decir, sus contactos directos). Proporcionamos evidencias anecdóticas sobre el significado que los individuos atribuyen a su red personal. Además de las reacciones espontáneas, recabamos información sobre las agrupaciones y los roles que identifican en el gráfico. En segundo lugar, comparamos de modo sistemático dos formas de representación de las redes personales: un gráfico que muestra los lazos fuertes entre los nodos que constituyen la red, y otro en el que destacamos el nivel de multiplicidad de los proveedores de ayuda.

En las siguientes páginas justificamos el estudio de los efectos de la visualización y presentamos el enfoque de experimentación llevado a cabo. Para ello proponemos un procedimiento de feedback visual que permite validar la descripción de la red personal con el informante.

\section{La percepción de las redes}

La información que proporciona un sociograma puede inducir a error. McGrath, Blythe y Krackhardt (1997) han demostrado que la disposición espacial de un grafo influye en la percepción de sus propiedades estructurales. Estos tres investigadores llevaron a cabo un experimento con 5 representaciones diferentes de una misma red, examinando las reacciones e interpretaciones de los participantes ante cada gráfico. Pese a tratarse de visualizaciones de los mismos datos, los observadores describían de modo diferente la centralidad, la intermediación y las agrupaciones de la red social en cada caso. Concretamente, la importancia atribuida a un actor tiende a aumentar cuando éste se sitúa en una posición más central en el gráfico, mientras que la cercanía entre grupos de actores suele estimular la identificación de agrupaciones.

Después de este primer trabajo empírico sobre los efectos de la visualización, se han llevado a cabo varios estudios que exploran el impacto de diferentes di-

${ }^{2}$ Para este último caso, sirve de ejemplo la aplicación de la técnica de netmirror en la dinámica de grupos (Borgatti \& Molina, 2002; Santolaya \& Maya Jariego, 2004). 
mensiones y propiedades de la representación gráfica. Por ejemplo, Brandes, Raab y Wagner (2001) han mostrado la utilidad de combinar indicadores de estatus social u organizacional con la representación de los lazos entre actores. Basándose en este enfoque, McGrath y Blythe (2004) estudiaron a continuación los efectos del movimiento y del formato de presentación de la red - con una distribución jerárquica o basada en la centralidad- en las interpretaciones por parte de los observadores.

En cualquier caso, la mayor parte de la investigación en este ámbito se ha centrado en mejorar la estética de diferentes formas de visualización con programas informáticos (véase una breve referencia en McGrath, Blythe y Krackhardt, 1997). Por lo general, se valora la claridad en la exposición de la información, evitando que los gráficos sean innecesariamente complejos. Cuando se introducen muchas dimensiones en la visualización es más fácil que se transmita información no deseada, dando lugar a interpretaciones erróneas. En un plano meramente estético, también se considera positivo que los nodos no estén demasiado cercanos entre sí, y que los lazos no sean excesivamente largos ni se superpongan unos con otros.

Las estrategias de visualización han sido menos estudiadas en el caso de las redes personales. Sin embargo, algunos trabajos recientes han evaluado el papel de la representación gráfica de las redes personales en la obtención de nueva información. Concretamente, la visualización de la red personal es una herramienta útil en las entrevistas cualitativas, y permite obtener del entrevistado agrupaciones más válidas y significativas que las que se obtienen de modo espontáneo en preguntas genéricas a priori (McCarty et al., 2004; Molina et al., 2005). Además, la visualización dinámica es en sí misma un procedimiento práctico para obtener información sobre las redes personales (McCarty y Govindaramanujam, 2005).

Como ya hemos adelantado, en este estudio intentamos comprender cómo interpretan los observadores la representación gráfica de sus redes personales, comparando sus reacciones ante dos estímulos diferentes. Por un lado, representamos los lazos fuertes entre los actores que componen su red personal. La idea de «lazos fuertes» remite a la frecuencia e intensidad de la relación, y ha sido ampliamente estudiada en el área del análisis de redes sociales (Granovetter, 1973, 1983). La representación de aquellas relaciones de mayor intensidad y frecuencia produce por lo general un gráfico más simple, que reduce la información al conjunto de contactos más significativos. En segundo lugar, elaboramos un gráfico donde se destaca la polivalencia de los nodos en la provisión de apoyo. La multiplicidad de tipos de ayuda es una noción habitualmente utilizada en la literatura sobre apoyo social (véase, por ejemplo, la revisión de Veiel y Baumann, 1992). Con este segundo gráfico, se muestra la ayuda percibida como disponible por el individuo, incluyendo información especialmente reveladora desde un punto de vista personal.

Se trata de dos estrategias diferentes para representar las relaciones más significativas para el entrevistado. En el primer caso la importancia de la relación 
es una característica de los lazos entre los actores; mientras que en el segundo gráfico la importancia de la relación se representa como una propiedad de los actores (es decir, de los nodos). Estamos comparando, además, una forma de visualización que subraya las propiedades estructurales de la red, con otra que destaca sin embargo los aspectos funcionales y de apoyo social.

En nuestro estudio, ambas formas de visualización son comparadas de modo sistemático en una serie de características que son relevantes según la literatura sobre los efectos de la visualización (McCarty et al., 2004; McGrath, Blythe y Krackhardt, 1997; McGrath y Blythe, 2004; Molina et al., 2005): se trata de la estética, la novedad, la utilidad, la revelación de contenidos íntimos, la detección de grupos, el reconocimiento de posiciones y la adecuada representación de la biografía personal, entre otras.

\section{MÉTODO}

Para llevar a cabo la investigación se siguieron tres pasos sucesivos. En primer lugar, entrevistamos a 208 estudiantes universitarios - como parte de un estudio de necesidades-, obteniendo información de sus redes personales. Con esa información, en segundo lugar, elaboramos tres representaciones gráficas de cada red personal, produciendo un total de 624 imágenes. Finalmente, 6 meses después de la primera entrevista, los gráficos fueron mostrados a un total de 173 encuestados, con el objetivo de (a) examinar sus reacciones en una entrevista cualitativa y (b) comparar dos formas de representación de las redes personales.

\section{Participantes}

En este estudio analizamos las redes personales de estudiantes universitarios que se desplazan casi a diario entre Alcalá y Sevilla. Se trata de un colectivo que desarrolla un estilo de vida metropolitano - a caballo de ambas localidades-, en el que es interesante evaluar los cambios en la estructura y la composición de sus redes personales asociados con dicha transición ecológica (véase, Maya Jariego, 2003).

Los 208 encuestados tienen un perfil socio-demográfico característico: se trata de una persona en torno a los veinte años de edad (23.26 años como promedio), soltera y que vive con sus padres. Han cursado una media de 4,1 años de estudios universitarios. Casi dos tercios de la muestra son mujeres y casi todos residen actualmente en Alcalá.

El universitario alcalareño realiza un promedio de 21.22 visitas mensuales a Sevilla (DT $=9.33$ ), con su desplazamiento de ida y vuelta en cada una de ellas. La gran mayoría va a Sevilla entre 5 y 7 veces por semana: el $40,4 \%$ lo hace a diario y el $42,8 \%$ de lunes a viernes. Una descripción más detallada de la muestra puede consultarse en Araya y Maya Jariego (2005) y en Maya Jariego et al. (2005). 


\section{Información sobre las redes personales}

Como primer paso, se obtuvo información sobre el núcleo de proveedores de apoyo (normalmente, entre 10 y 15 personas). A continuación se solicitó a los encuestados que completaran el listado hasta nombrar un total de 25 alteri. Finalmente, procedimos a la evaluación de lazos entre pares $(25 \times 25)$, obteniendo también información atributiva sobre «el tipo de relación», el «lugar de residencia» y el «medio habitual de comunicación» de cada alteri.

El generador de nombres utilizado fue el Arizona Social Support Interview Schedule (ASSIS) (Barrera, 1980). Se trata de una entrevista semi-estructurada que permite obtener los nombres de las personas con las que el encuestado cuenta para tratar sus asuntos personales, pedir dinero prestado, solicitar consejo, obtener feedback positivo, conseguir ayuda instrumental y compartir el tiempo libre. Es un conjunto de seis preguntas que generan aproximadamente entre 5 y 15 nombres de proveedores de apoyo (lazos fuertes tales como la pareja, los parientes y amigos muy cercanos). Los entrevistados tienen la opción de mencionar apodos, iniciales o cualquier sistema de identificación en caso de que no quieran utilizar nombres reales.

Posteriormente se les pidio completar una lista hasta llegar a 25 nombres. Para completar el listado se les preguntó por personas con las que han tenido contacto en el último tiempo, independientemente de que fueran relaciones muy cercanas o no. Con este procedimiento se obtienen nombres de personas con las que por lo general se tiene una relación más débil que con aquellos mencionados en el cuestionario de apoyo.

Con esta información, construimos 208 matrices de redes personales (de $25 \times 25$ actores) que se refieren a 5.200 relaciones en total (208 egos $\times 25$ alteri). Cada una de estas matrices fue manejada y analizada con UCINET 6.0 (Borgatti, Everett y Freeman, 2002).

\section{Representación gráfica}

Se utilizaron tres dimensiones para representar las redes personales, utilizando para ello el color, el tamaño del nodo y la presencia o ausencia de relaciones:

- Composición. Los nodos azules (más oscuros) representan a individuos residentes en Alcalá, los nodos rojos (más claros) corresponden a personas asentadas en Sevilla y los nodos negros simbolizan a los que viven en otras ciudades. Esta dimensión no forma parte de los criterios de comparación que hemos presentado más arriba, sino que se refiere a los aspectos sustantivos del estudio (véase, Maya Jariego et al., 2005).

- Multiplicidad de los proveedores. El tamaño del nodo representa el número de tipos de ayuda que proporciona cada proveedor, de acuerdo con la información obtenida en el ASSIS. 
- Fortaleza de la relación. Se establecieron tres niveles diferentes: 0 , «A y B no se conocen»; 1, «A y B se conocen»; y 2 , «A y B se conocen muy bien, tienen bastante relación». Gráficamente, la existencia o no de lazos puede establecerse de acuerdo con los distintos niveles de fortaleza de la relación.

Para someterlo a la evaluación del impacto, desarrollamos dos formas diferentes de representacion visual ${ }^{3}$ :

- Por un lado representamos la red personal del encuestado para los lazos fuertes. Es decir, sólo mostramos las relaciones de nivel 2 entre los nodos que componen la red personal. Es el Gráfico Tipo I.

- Por otro lado, representamos la red personal reflejando la importancia relativa de cada nodo en calidad de proveedores de apoyo. En este caso representamos las relaciones de nivel 1 entre los nodos, reflejando con el tamaño la multiplicidad de cada vínculo. Es el Gráfico Tipo II.

\begin{tabular}{lll|}
$\begin{array}{l}\text { Gráfico Tipo I. } \\
\text { Lazos fuertes (valor 2 de relación) entre } \\
\text { los 25 contactos de la red personal del } \\
\text { entrevistado. }\end{array}$ & $\begin{array}{l}\text { Gráfico Tipo II. } \\
\text { Proveedores múltiples que destacan en la } \\
\text { red de conocidos (valor 1 de relación) del } \\
\text { entrevistado. }\end{array}$
\end{tabular}

\section{La entrevista de feedback}

El cuestionario de feedback se aplicó a un total de 173 personas. Ambos tipos de gráficos fueron presentados a cada encuestado (alterando el orden de presentación en la mitad de los casos). Durante la entrevista se pidió a los estudiantes que comentaran las imágenes de su red personal.

${ }^{3}$ Un tercer gráfico, no sometido a comparación pero que fue utilizado durante la entrevista, representaba las relaciones de nivel 1 («se conocen»), sin modificar la fortaleza del lazo ni la multiplicidad del proveedor de ayuda. 
Los entrevistados expresaron inicialmente sus impresiones de modo abierto. A continuación se formularon preguntas que sirvieron de guía para el comentario de la red personal, con cuestiones como las siguientes: describir el gráfico; dividir el grafo en conjuntos de nodos; identificar actores clave en la red personal; informar de errores, etcétera.

Para terminar, se informó a los encuestados de la forma en la que se habían construido los gráficos Tipo I y Tipo II. Seguidamente, ambos fueron comparados en un listado de 11 características que fueron puntuadas con una escala tipo Likert de 1 a 5 (por un total de 170 participantes). Dicha información fue manejada y analizada con SPSS para Windows 13.0, junto con el resto de datos obtenidos en la encuesta.

\section{RESULTADOS}

En este apartado (a) presentamos el proceso de entrevista con un caso de aplicación, (b) relatamos las impresiones cualitativas de los observadores y (c) comparamos de modo sistemático los dos modos de representación gráfica. En primer lugar, el ejemplo de aplicación puede servir para ilustrar cómo se desarrolla el feedback cualitativo con los informantes.

\section{Un caso de aplicación del feedback}

La entrevista sigue una secuencia de dos gráficos que se utilizan como estímulo para suscitar los comentarios del entrevistado. A partir de una lista de preguntas y del diálogo con el observador, se va generando una descripción significativa de la red personal en cuestión. Veamos un ejemplo en el que hemos seleccionado y organizado los comentarios de una chica universitaria de 25 años: Fermina.

En este caso se presenta en primer lugar el Gráfico I, que muestra sólo las relaciones de nivel 2 - es decir, los «lazos fuertes»- entre los 25 actores que componen su red. En la imagen hemos dibujado las agrupaciones que realiza Fermina a lo largo del proceso de entrevista: «Tengo una red amplia, en la que hay tres círculos diferentes, que no están relacionados entre sí. Son la gente de mi trabajo, la gente de mi segundo trabajo, mi familia y mis amigos de Alcalá. Entre sí no se conocen: son tres grupos que no están relacionados. Mi familia está por delante de mis amigos, conoce a mis amigos. Y mi novio es el único que conoce a gente de los tres grupos. Es la persona más destacada porque puede intercambiar con todos».

A continuación se presenta el Gráfico II, que muestra mayor rango de relaciones (el nivel 1 y superiores) y representa con el tamaño del nodo el grado de polivalencia de los diferentes proveedores de apoyo. La entrevistada dispone en este caso de la referencia del primer gráfico, ya comentado: «Aquí todo el mundo 


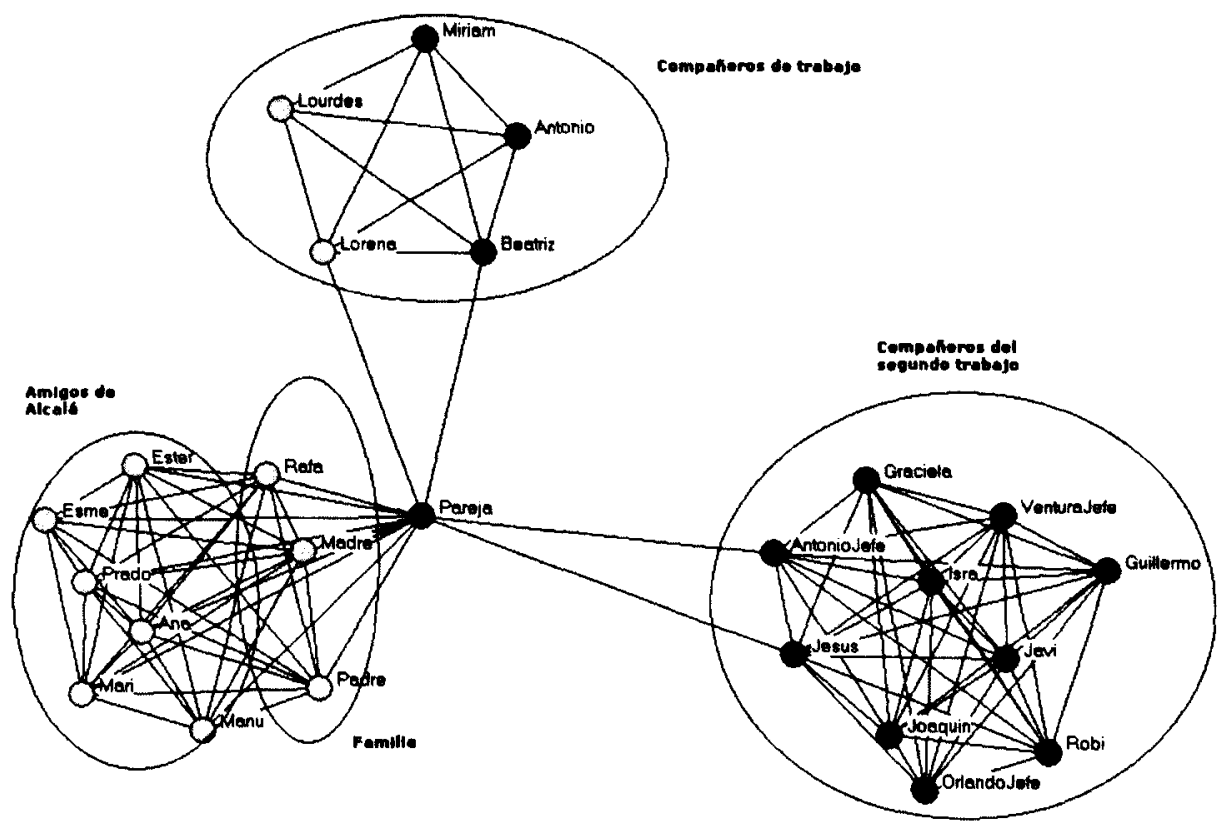

Grafico 1. «Lazos fuertes» de Fermina.

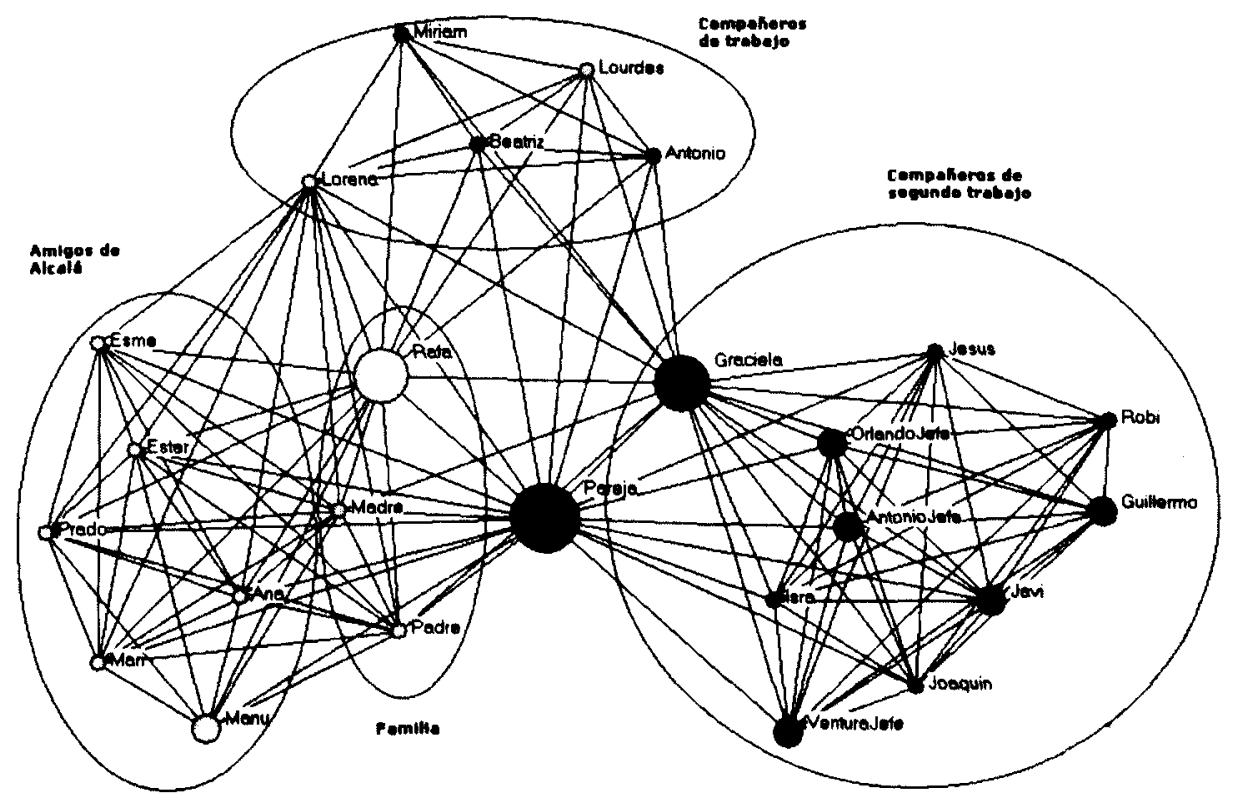

Gráfico 2. «Multiplicidad de apoyo de los proveedores» de Fermina. 
conoce a casi todos. Se aprecian más interrelaciones. Me gusta más porque parece más real. Hay tres personas especialmente importantes, que tienen más relación con el resto. Son mi novio, mi amiga Graciela y mi hermano Rafa. Pero yo haría las mismas agrupaciones que en el caso anterior. Los mismos cuatro grupos».

Como vemos, la red personal de Fermina se organiza en espacios sociales claramente delimitados: la familia, los amigos y los lugares de trabajo. A su vez, dichos espacios están visiblemente distribuidos entre Sevilla y Alcalá ${ }^{4}$, correspondiendo, respectivamente, a los ámbitos laboral y social-afectivo. La pareja ocupa una posición destacada, siendo la figura con los mayores niveles de centralidad y potencial de intermediación. Sin embargo, hay otros actores clave en la red personal, como el hermano en el entorno alcalareño y una compañera de trabajo en Sevilla. Como muestra este ejemplo, el Gráfico Tipo I representa con más claridad las propiedades estructurales de la red personal. Sin embargo, la encuestada prefiere en este caso la imagen que refleja mayor conectividad social (el Gráfico Tipo II).

\section{Reacción e interpretaciones de las redes personales}

Los encuestados atribuyen en general validez a la información representada gráficamente en su red personal. En su mayoría se muestran muy interesados durante la conversación, y participan activamente en el análisis de las propiedades de ambos gráficos. Con la entrevista cualitativa, los participantes pueden dotar de significado a su red personal: sugiriendo agrupaciones; informando de los espacios sociales en los que participan; aclarando el rol de los actores más importantes en la estructura de relaciones, etcétera. También aluden en ocasiones al tipo de red que prefieren o a las estrategias que dicen seguir en sus relaciones con los demás: por ejemplo, unos optan por la integración de grupos e individuos mientras que otros prefieren la distribución en espacios sociales delimitados... Pero veamos de forma organizada los criterios que siguen para interpretar la representación gráfica de las redes personales.

\section{Claves en la descripción subjetiva de las redes personales}

Cuando comentan sus redes personales, los encuestados siguen una pauta caracterizada por (a) la organización en una estructura de círculos concéntricos, (b) el reconocimiento de agrupaciones y (c) la ordenación según la importancia relativa de los nodos.

La mayoría de los entrevistados procede describiendo el gráfico de su red personal desde el centro hacia la periferia. Situando a ego en un centro hipotético, la

${ }^{4}$ Los nodos de color más claro (amarillo en el original) corresponden a conocidos alcalareños, mientras que los contactos con sevillanos se representan con el color más oscuro (en azul). 
información se organiza en círculos concéntricos. Los comentarios combinan la cercanía percibida por ego con la distancia entre grupos. Por eso es frecuente que se comience por la familia y el grupo de amigos más íntimos, para pasar a continuación a la localización de otras agrupaciones. No obstante, esta secuencia desde dentro hacia fuera se produce con independencia de las categorías identificadas por el encuestado, y aunque el gráfico muestre una estructura dispersa.

«Es como una cebolla. En el núcleo los amigos más fuertes, y a partir de ahí salen los conocidos. Con los del perímetro de fuera hay menos contacto y a su vez menos contacto entre ellos. Es una red cerrada, cohesionada y estable. Y yo la cuido manteniendo el contacto» (Caso 143).

«Por un lado está la gente de mi entomo, la más cercana a mí, que son sobre todo gente de Alcalá. Y por otro lado dos grupos más separados... la vida personal y la vida profesional. Hay mucha relación entre la gente más cercana a mí y menos entre los demás» (Caso 148).

«Tanto en un gráfico como en el otro, los principales están en el centro» (Caso 6).

«Es una cebolla, por capas, de la más íntima a la menos íntima» (Caso 208).

Una reacción habitual consiste en la búsqueda de agrupaciones. Los encuestados describen la estructura de su red identificando los grupos más significativos, y las relaciones entre los mismos. Esta tendencia a dividir la red en subconjuntos se observa incluso antes de que en la entrevista se les pida explícitamente que señalen los grupos existentes en su red personal.

Después de reconocer las agrupaciones más destacadas, los encuestados suelen buscar personas clave en cada grupo de relación. Finalmente, la cercanía entre grupos también es una referencia visual importante.

«Esta es mi familia, estos son mis amigos de la facultad y estos son mis amigos de Sevilla... Mis amigos de Sevilla y la familia están muy relacionados. Sin embargo, la gente de la facultad está algo aparte» (Caso 62).

«Laura es la más importante en mi grupo de la Facultad, y es la que une a ese grupo con la gente de Alcalá. Carlos es mi mejor amigo en Alcalá. Y Javier es la persona más destacada en el grupo de Dos Hermanas» (Caso 13).

"Veo cómo de cada grupo destaca una persona. Pili en el caso de Alcalá, Isabel en el caso de la familia y Joaquín en el caso de la facultad» (Caso 60).

«Cecilia en el grupo de mi facultad... del grupo de Sevilla destaca María... y arriba Ángela» (Caso 62).

De las tres dimensiones utilizadas en la representación gráfica -composición, fortaleza del vínculo y multiplicidad-, es la polivalencia de los provee- 
dores de ayuda la más destacada. El tamaño del nodo se interpreta inmediatamente en términos de importancia relativa de los actores que proporcionan apoyo. En cambio, muchos encuestados no llegan a reconocer de modo espontáneo que el color simboliza el lugar de residencia de cada alteri. En el caso de la representación de los lazos fuertes, la mayoría de las veces es necesario explicar al entrevistado el procedimiento por el que se ha elaborado esa forma de visualización de su red personal (el gráfico Tipo I).

Por otro lado, el tamaño del nodo parece inducir una clasificación en función de la intensidad de relación (disponiendo a grupos e individuos de mayor a menor). No obstante, ese ranking de actores se organiza según la polivalencia de los proveedores, y resulta menos saliente la importancia relativa en términos de centralidad o de intermediación, entre otros indicadores relacionales.

«Mi madre, mi hermana, mi padre... Por orden de importancia, iqué curioso!» (Caso 20).

«Este es el grupo de mis amigos íntimos, luego los amigos más lejanos y luego los conocidos» (Caso 158).

Para el grueso de los participantes resulta gratificante visualizar su propia red personal. Parece proporcionarles información significativa y, en algunos casos, sorprendente.

\section{Algunas propiedades y fenómenos estructurales}

Los encuestados varían considerablemente en su capacidad de interpretación y análisis de la red personal. Pero en su conjunto realizan una descripción que permite abstraer algunas características compartidas. Por ejemplo, los encuestados se refieren al núcleo de su red como el componente más estable a lo largo del tiempo; informan de los cambios experimentados desde que tuvo lugar la primera entrevista; y señalan el papel destacado que generalmente tiene la pareja o algún vínculo confidente.

El núcleo suele estar constituido por un pequeño número de familiares y amigos íntimos. La familia, los contactos alcalareños y, en general, los grupos con los que se ha mantenido durante largo tiempo la relación, muestran mayor nivel de apiñamiento que aquellas relaciones más recientes. Los encuestados ponen inmediatamente de manifiesto los cambios que ha experimentado su red desde que cumplimentaron el cuestionario seis meses atrás.

«A Ah! ¡Pero esto ha cambiado! Con Zoraida, que era muy buena amiga, no me hablo y ya no está en la red, y con Patricia me he peleado» (Caso 19).

«Javier ya se ha ido, ya no tengo contacto con él. Fernando está de Erasmus en Francia. Pilar se ha ido también, pero no sé donde...» (Caso 37). 
«Ahora en la universidad conozco más gente, y pondría a un grupo de la facultad que no son de Alcalá» (Caso 64).

Del conjunto de actores suele destacar la posición de la pareja, en el caso de aquellos que tienen novio, están casados, o disponen de un vínculo confidente. Se trata de un actor que, por lo general, es polivalente en términos de apoyo, ocupa una posición central en la estructura de relaciones, hace muchas veces de mediador entre grupos y aporta relaciones al encuestado.

Por eso son especialmente significativos los cambios que pueden producirse en la organización de la red personal como consecuencia de la ruptura de la relación con la pareja. En la muestra, de 23 años de edad como promedio, este tipo de cambios parecen relativamente frecuentes. Concretamente, 11 encuestados (el 6.4 por ciento) informan al analizar su red de la ruptura con quien era su pareja en el momento de la primera entrevista.

«Las personas más allegadas a mí están más cerca de mi novio» (Caso 16).

«Estos de aquí [14 nodos] son personas que conozco desde que estoy con mi novio» (Caso 16).

«Mi novio es el centro que une los dos grupos, porque los puntos rojos son los amigos de mi novio" (Caso 42).

«Está claro que mi novia es el nexo de unión entre varios grupos, ¿no?» (Caso 153).

«Hay dos grupos... y en el centro está mi novio. Un grupo es la familia y el otro son amigos y conocidos de la facultad, y mi novio conoce a todo el mundo" (Caso 111).

\section{Los lazos fuertes frente a la multiplicidad de los proveedores de ayuda}

Los dos tipos de representación producen reacciones ligeramente diferentes. De acuerdo con los comentarios que hacen los participantes, parece que el gráfico de «lazos fuertes» (Tipo I) suscita con más frecuencia la formulación de agrupaciones, mientras que el gráfico basado en la «multiplicidad de apoyo» (Tipo II) tiende a generar descripciones que se organizan en función de la importancia relativa de los nodos. Vemos un ejemplo a continuación, aunque volveremos sobre este punto en próximos apartados:

- Gráfico Tipo I: «Destaca el núcleo reducido familiar. Y luego hay dos grupos de amigos: los de Alcalá y los de la facultad. Mis amigos no se conocen entre ellos, los únicos que están relacionados son los miembros de mi familia... yo soy la única conexión entre muchos de mis amigos» (Caso 2). 
- Gráfico Tipo II: «Samuel y Mónica son pilares en mi red. Samuel es mi novio y Mónica es mi mejor amiga. Es como si fueran de mi familia. Son los más importantes. Y a través de personas que yo creo importantes he conocido a otras. Ellos conocen además mi círculo más cercano» (Caso 2).

La representación de los lazos fuertes muestra con mayor nitidez los espacios sociales en los que participan los encuestados. Quizá esa es la razón por la que, con más frecuencia, recurren a la comparación entre el lugar de estudios y la ciudad de residencia. También hace más probable los comentarios sobre la estructura de la red y su influencia en las relaciones interpersonales. Los siguientes comentarios están referidos al Gráfico Tipo I:

«Tengo como dos círculos. Los amigos de Alcalá y los de Sevilla. Y tienen pocas relaciones entre sí» (Caso 6).

«En este gráfico se ven claramente las relaciones que hay entre los grupos» (Caso 13).

«Te permite distinguir una relación más individual, de persona a persona, con los que aparecen como puntos sueltos, y más grupal con los otros» (Caso 2).

«Estos son los amigos del Instituto de mi marido, que ahora son también mis amigos porque nos vemos con ellos». «En este gráfico veo claramente la parte de mis amigos que comparten además profesión conmigo. Es el grupo de "amigos-profesionales". Son el centro de mi red, y están entre los amigos y los profesionales. ¿Ves que antes no se veía?» (Caso 161).

«Este gráfico [Tipo I] muestra la relación que tiene cada uno entre ellos, mientras que el otro [Tipo II] muestra más la importancia que cada uno tiene para mí» (Caso 53).

A veces genera información novedosa o sorprendente, porque algunos nodos ocupan una posición relevante, pese a no destacar por su relación con el entrevistado:

«Es verdad. Isaac tiene una posición destacada. Es el único que conoce a gente de mi trabajo, a mis amigos y a mi novia. Está en un sitio clave en mi red, pero no tengo una relación especial con él» (Caso 156).

«Con Rafa no tengo mucha relación, y sin embargo fíjate que está en el centro, y conoce a gente de todos los grupos. Pero no tengo mucha relación con él» (Caso 177).

Por comparación, el gráfico que representa la multiplicidad de ayuda resulta más intuitivo, puesto que destaca a las personas más significativas para el entrevistado. Se trata de una imagen que representa con claridad el núcleo de su red y, por lo general, de fácil interpretación. 


\section{Errores}

La entrevista permite identificar errores en la obtención de información, en el tratamiento de los datos o en la representación gráfica de las redes personales. Concretamente, 28 entrevistados (el 16,18 por ciento) mencionaron algún tipo de error en la representación de su red personal. Las correcciones más frecuentes consistieron en la eliminación de algún enlace que realmente no existía entre un par de nodos (en 12 casos) o la identificación de una asignación equivocada de un nodo a Sevilla o Alcalá (en 10 casos). Teniendo en cuenta que los entrevistados informaron sobre un total de 4.325 nodos y sus relaciones se trata de una incidencia de errores mínima ${ }^{5}$.

Tabla 1

Errores informados por los encuestados en la visualización de su red personal

\begin{tabular}{|l|r|c|}
\hline & \multicolumn{1}{|c|}{} & \% sobre el total de nodos/relaciones \\
\hline Enlaces entre nodos* & 16 & 0.031 \\
Composición (Sevilla/Alcalá) & 10 & 0.23 \\
Intensidad de relación $(0,1,2)$ & 1 & 0.002 \\
Un actor aparece dos veces & 1 & 0.023 \\
\hline
\end{tabular}

* En 12 casos identifican un lazo entre dos nodos que realmente no debería existir; y en 4 casos detectan que falta una relación que debería estar presente entre dos nodos.

\section{Comparación entre los dos tipos de representación gráfica}

Después de realizar una entrevista cualitativa valorando cada gráfico, le pedíamos al entrevistado que los valorasen en un listado de dimensiones. La comparación entre ambos, aplicando la $t$ de Student para muestras relacionadas está resumida en la Tabla 2.

En su conjunto, los entrevistados muestran preferencia por el gráfico que representa la polivalencia de los proveedores de ayuda, destacados de entre el resto de relaciones. De un modo más concreto, el Gráfico tipo II proporciona información más novedosa para los entrevistados; permite identificar mejor a las actores clave en la red personal; refleja adecuadamente la importancia de los alteri; y muestra más fielmente la posición que ocupa el encuestado en ese conjunto de relaciones.

El Gráfico tipo I, que representa los lazos fuertes, sólo destaca por su utilidad para identificar agrupaciones en la red personal. Es decir, sirve para definir los grupos (o espacios sociales) entre los que se mueve el entrevistado.

${ }^{3}$ En cada red personal se evalúan un total de 300 pares de nodos, por lo que para los 173 entrevistados en el feedback examinamos un total de 51.900 lazos potenciales. 
Tabla 2

Comparación de los dos sistemas de representación visual $(n=170)$

\begin{tabular}{|c|c|c|c|c|c|c|}
\hline \multirow{2}{*}{ Dimensiones } & \multicolumn{2}{|c|}{ Gráfico I } & \multicolumn{2}{|c|}{ Gráfico II } & \multirow{2}{*}{$t$ de Student ${ }_{(\mathrm{gl}=1,169)}$} & \multirow{2}{*}{ Sig. } \\
\hline & $\mathbf{M}$ & DT & $\mathbf{M}$ & DT & & \\
\hline Estética & 3,35 & 1.01 & 3,17 & 1.15 & 1.55 & 0.121 \\
\hline Agrupaciones & 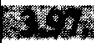 & 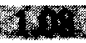 & 3.50 & 1.11 & 4.31 & 0.001 \\
\hline Personas clave & 3.14 & 1.25 & Exts & metas & -9.00 & 0.001 \\
\hline Novedad & 2.05 & 1.17 & & 2tid & -3.59 & 0.001 \\
\hline Biografía & 3.47 & 1.14 & 3.55 & 1.14 & -1.10 & 0.271 \\
\hline Posición de ego & 3.08 & 1.35 & 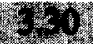 & 153 & -2.56 & 0.011 \\
\hline Importancia de los alteri & 2.93 & 1.25 & 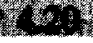 & nom & -11.40 & 0.001 \\
\hline Relación Alcalá/Sevilla & 3.50 & 1.52 & 3.41 & 1.50 & 1.49 & 0.136 \\
\hline Utilidad & 2.70 & 1.26 & 2.80 & 1.27 & -1.32 & 0.186 \\
\hline Intimidad & 1.76 & 1.15 & 1.88 & 1.25 & -1.80 & 0.073 \\
\hline Valoración global & 3.83 & 1.04 & tout & sates & -2.51 & 0.013 \\
\hline
\end{tabular}

No se observan diferencias entre ambos sistemas de representación en la estética, el grado de intimidad de la información, la capacidad para representar la biografía personal, la posición del entrevistado en la relación entre Alcalá y Sevilla, ni en la utilidad de la información que proporciona.

\section{Categorías y agrupaciones}

Los participantes utilizan en promedio 4.05 categorías en la identificación de agrupaciones en los gráficos, con un rango que oscila entre 0 y 8 . No se observaron diferencias significativas en el número de categorías aplicadas en cada gráfico.

La forma más habitual de agrupación consiste en identificar grupos naturales. Se trata por lo general de conjuntos bien definidos y homogéneos, designados con categorías tales como «la familia», «los amigos», «los compañeros de trabajo», «los compañeros de la facultad», «la pareja», etcétera. Un caso particular menos frecuente en este tipo de agrupaciones - concretamente, el 3,4 por ciento en cada gráfico- es la clasificación por lugar de residencia: «la gente de Alcala», «la gente de Sevilla», etc.

La segunda estrategia de categorización consiste en agrupar a los actores de acuerdo con el grado de intensidad o cercanía en la relación con el entrevistado. En este caso suelen identificarse, por ejemplo, a «amigos íntimos» frente a "gente sólo conocida» o "personas que conozco indirectamente, a través de otros». Este tipo de descripción aparece frecuentemente en combinación con la anterior, de localización de grupos naturales. 
Como se observa en la Tabla 3, los encuestados tienden a aplicar el mismo sistema de clasificación en ambos gráficos, existiendo una asociación significativa en el tipo de categorías utilizada en cada caso (Chi-cuadrado $=118.370$, $\mathrm{p}<.0 .0001$ ). Sin embargo, la agrupación por niveles de intensidad es claramente más frecuente en la representación de la multiplicidad del apoyo social. Concretamente, en el Gráfico Tipo II hay un 10,37 por ciento más de casos de categorización por intensidad, y un 6,71 por ciento menos de clasificación por grupos naturales.

Tabla 3

Categorías aplicadas en la interpretación de ambos gráficos $(n=164)$

\begin{tabular}{|c|c|c|c|c|c|c|}
\hline & \multicolumn{2}{|c|}{ Grafico I } & \multicolumn{2}{|c|}{ Gráfico II } & \multirow{2}{*}{$t$ de Student $\left.{ }_{(\mathrm{gl}}=1,163\right)$} & \multirow{2}{*}{ Sig. } \\
\hline & $\mathbf{M}$ & DT & $\mathbf{M}$ & DT & & \\
\hline Número de categorías & 4.02 & 1.29 & 4.09 & 1.42 & -0.716 & 0.47 \\
\hline Tipo de agrupaciones & $\mathrm{F}$ & $\%$ & $\mathrm{~F}$ & $\%$ & Chi-cuadrado $_{(\mathrm{gl}=1,9)}$ & Sig. \\
\hline $\begin{array}{l}\text { Por grupos } \\
\text { Por intensidad de relación } \\
\text { Mixta (grupos e intensidad) } \\
\text { Otras categorizaciones }\end{array}$ & $\begin{array}{r}93 \\
9 \\
60 \\
2\end{array}$ & $\begin{array}{r}56.7 \\
5.5 \\
36.6 \\
1.2\end{array}$ & $\begin{array}{r}75 \\
19 \\
67 \\
3\end{array}$ & $\begin{array}{r}45.7 \\
11.6 \\
40.9 \\
1.8\end{array}$ & 118.370 & 0.0001 \\
\hline
\end{tabular}

Efectos del orden de presentación y validación de los resultados

Cabe la posibilidad de que los encuestados aprendan a interpretar los gráficos en la aplicación de los dos estímulos consecutivos. Puede haber casos en los que se transfiera información o hipótesis de una visualización a la siguiente. Para controlar el posible efecto del orden de presentación, alternamos la secuencia para la mitad de los participantes. En los análisis posteriores no encontramos ningún efecto del orden en los resultados. Para comprobarlo, realizamos dos series de comparaciones de medias (para los gráficos I y II) en el listado de dimensiones de la Tabla 2, no obteniendo ninguna diferencia significativa. Tampoco se observaron diferencias en el tipo de categorización según el orden de presentación (con Chi-Cuadrado de 2.15 y p $<.53$, para el gráfico I; y Chi-Cuadrado de 3.67 y $\mathrm{p}<.299$, para el gráfico II).

Por otro lado, no se observó relación significativa entre las propiedades estructurales de las redes personales (es decir, los valores promedios de degree, closseness, betweeness y eigenvector centrality) y las puntuaciones atribuidas a cada gráfico. Tampoco las variables demográficas (género, edad, estado civil y tipo de estudios universitarios) parecen tener un impacto significativo en las estrategias para interpretar los gráficos. Tan sólo merece la pena destacar el hecho de que las mujeres tienden a considerar más novedosa la información que le pro- 
porciona la visualización de su red personal que los hombres, tanto en el gráfico I $\left(t_{(g l=1,168)}=-2.2, p<.033\right)$ como en el gráfico II $\left(t_{(g l=1,168)}=-2.9, p<.004\right)$.

\section{DISCUSIÓN}

Las técnicas de visualización constituyen una poderosa herramienta para explorar los datos de redes sociales y para comunicar sus propiedades estructurales. En esta investigación hemos partido del supuesto de que es necesario comprender cómo perciben las representaciones gráficas los destinatarios de la misma, para potenciar la efectividad de su aplicación. El feedback de las redes personales nos ha permitido comprobar precisamente la dinámica de procesamiento activo que pone en marcha el dispositivo visual en los receptores de la información.

Los gráficos de redes ego-céntricas proporcionan una base objetiva para discutir sobre las relaciones interpersonales del encuestado. En la entrevista cualitativa permite completar información y darle significado a la representación de la red personal. Como hemos mostrado, el encuestado puede hacer agrupaciones, explicar el papel de algunos actores y aportar datos biográficos con los que interpretar los resultados. De ese modo, se obtiene una descripción en profundidad de la red personal, que difícilmente puede conseguirse sin la recogida sistemática de información y la devolución posterior de los resultados a través de la representación visual.

En este estudio hemos comparado dos formas diferentes de representar «lazos fuertes». Los lazos fuertes pueden ser definidos por la intensidad de la relación o por la polivalencia en términos de apoyo de cada alteri. De acuerdo con los resultados, esta segunda alternativa -que hemos representado con el Gráfico Tipo II- resulta más significativa para la percepción de los entrevistados. Los datos permiten suponer que el apoyo social es una característica más saliente en la percepción de los observadores que las propiedades estructurales de la red personal. La enumeración de proveedores polivalentes es una forma simple y selectiva de reflejar las funciones de apoyo, mientras que la interpretación de los lazos requiere de un modo de proceder más analítico y sistematizado. No obstante lo anterior, la visualización selectiva de las relaciones de mayor intensidad -que hemos representado con el Gráfico Tipo I- hace más fácil la identificación de grupos claros en la red personal del encuestado.

Para describir las redes, los observadores suelen seguir una estrategia que combina algún criterio implícito de prelación entre actores o entre relaciones con la detección de agrupaciones significativas. También se observan diferencias aparentemente asociadas con el tipo de gráfico comentado. Concretamente, la representación de «lazos fuertes» parece inducir una descripción más basada en grupos y espacios sociales; mientras que la representación de la multiplicidad de los proveedores de ayuda induce una descripción basada en conjuntos de diferentes niveles de «importancia». 
Sin embargo, nuestro estudio no es de carácter concluyente. Por eso sería de interés sondear si las diferencias observadas entre gráficos corresponden al desigual impacto de la visualización del apoyo social y de las propiedades estructurales, o más bien se relaciona con el propio formato de representación. No en vano hay un tipo de gráfico en el que la fortaleza del lazo se presenta como una propiedad de los nodos (la multiplicidad de ayuda), mientras que en el otro se trata de una propiedad de las relaciones (en términos de lazos fuertes).

A partir de nuestro estudio pueden derivarse una serie de recomendaciones prácticas para el diseño de gráficos de redes. Aunque los resultados son exploratorios y deben ser interpretados con cautela, parece que los lazos fuertes y la multiplicidad de los proveedores son dos dimensiones particularmente relevantes en la descripción de las redes egocéntricas. La combinación de ambas dimensiones reúne a priori la significación personal del apoyo social con la potencialidad para identificar grupos a través de los lazos fuertes.

\section{AGRADECIMIENTOS}

Este artículo se ha beneficiado de los comentarios de Carlos Lozares, José Luis Molina y Laura Teves. En las entrevistas participaron activamente Esperanza Vergara, María José Expósito y Pilar de la Iglesia. Estamos en deuda con los universitarios alcalareños que ofrecieron amablemente su tiempo para participar en la entrevista. La investigación fue posible gracias al apoyo de Jesús Téllez, Marcelino Colete y Susana Postigo. 


\section{REFERENCIAS BIBLIOGRÁFICAS}

ARAYA DuIISIN, R. y MAYA JARIEGo, I. (2005): «Los puentes interlocales: las redes personales de los universitarios alcalareños en Sevilla». En: Porras, J. I. y Espinoza, V. (Eds.). Enfoques y aplicaciones del análisis de redes sociales. Universidad Santiago de Chile y Universidad Bolivariana. Santiago de Chile, Chile.

Borgatti, S. y Molina, J. L. (2002); «What Can Social Network Analysis Do for Management Consulting». «Building Effective Networks» Conference, Academy of Management, Denver, Colorado, 9 a 14 de agosto.

BRANDES, U.; RAAB, J. y WAGNER, D. (2001): «Exploratory network visualization: simultaneous display of actor status and connections». Journal of Social Structure, 2, \#4.

FREEMAN, L. C. (2005): «Graphic techniques for exploring social network data». En: P. J. Carrington, J. Scott y S. Wasserman (Eds.). Models and methods in social network analysis. New York: Cambridge University Press.

- (2004): The development of social network analysis. A study in the sociology of science. Vancouver, BC: Empirical Press.

- (2000): «Visualizing social networks». Journal of Social Structure, 1, \#1.

GRANOVETTER, M. S. (1973): «The strength of weak ties». American Journal of Sociology, 78, pp. 1360-1380.

- (1983): «The strength of weak ties: a network theory revisited». Sociological Theory, 1, pp. 203-233.

MAYA JARIEGo, I.; ARAYA, R. y ARMitage, N. (2005): «Redes personales y sentido de comunidad de los universitarios alcalareños en Sevilla: distribución del tiempo y espacios sociales». III Seminario para el análisis de redes sociales en Sevilla: International workshop on network visualization, 7 a 9 de septiembre de 2005.

Maya Jariego, I.; Araya, R.; Armitage, N.; Holgado, D. y Vergara, E. (2005): Participación ciudadana y sentido de comunidad en Alcalá de Guadaíra: la implicación comunitaria de los universitarios alcalareños. Laboratorio de Redes Personales y Comunidades, Universidad de Sevilla. Estudio patrocinado por el Ayuntamiento de Alcalá de Guadaíra.

MAYA JARIEGO, I. (2003): «A general typology of personal networks of immigrants with less than 10 years living in Spain». XXIII International Sunbelt Social Network Conference. Cancún (México).

MCCARTY, C. y Govindaramanujam, S. (2005): A modified elicitation of personal networks using dynamic visualization. Connections, 26 (2), pp. 61-69.

McCarTy, C.; Molina, J. L.; AgUILAR, C. y ROTA, L. (2004): Personal network visualization as an aid for qualitative interviews.

MCGRATH, C. y BLYTHE, J. (2004): «Do you see what I want you to see? The effects of motion and spatial layout on viewers' perceptions of graph structure». Journal of Social Structure, 5, \#2.

MCGRATH, C.; BLYTHE, J. y KRACKhARDT, D. (1997): The effect of spatial arrangement on judgments and errors in interpreting graphs. Social Networks, 19, pp. 223-242.

MOLINA, J. L.; MCCARTY, C.; AGULAR, C. y ROTA, L. (2005): «La estructura social de la memoria». Empiria.

SANTOLAYA, F. J. y MAYA JARIEGO, I. (2004): «Comunidades virtuales y acción voluntaria: la constitución de la lista de distribución de correo electrónico E-Voluntas». XVIII IAVE World Volunteer Conference. Barcelona, 17 a 21 de agosto de 2004.

VEIEL, H. O. F. y BAUMANN, U. (Eds.) (1992): The meaning and measurement of social support. New York, Hemisphere Publishing. 


\title{
RESUMEN
}

En este artículo examinamos el impacto de dos formas diferentes de representación visual de las redes personales de 208 universitarios en la percepción de los observadores. Siguiendo un enfoque de experimentación, comparamos las reacciones e interpretaciones de 173 encuestados al observar gráficos de sus redes personales que representan (a) los lazos fuertes o (b) la multiplicidad de tipos de ayuda de un conjunto de 25 actores de su entorno personal. Los participantes mostraron preferencia por el gráfico que subraya las funciones de apoyo social de su red. A juicio de los entrevistados, el gráfico que representa la multiplicidad de los proveedores de apoyo social facilita la identificación de actores clave, aporta información más novedosa, refleja adecuadamente la posición de ego en la estructura de relaciones y es, en general, una mejor representación de su red personal. Sin embargo, la representación de los lazos fuertes facilita la identificación de agrupaciones en la red personal. Basándonos en los resultados, discutimos la pertinencia de este método de exploración, elicitación y validación de las redes personales de los encuestados.

\section{PALABRAS CLAVE}

Redes Personales, Representación Gráfica, Lazos Fuertes, Multiplicidad del Apoyo Social.

\begin{abstract}
In this study, we explore the effects of two ways of visualization on viewers' perceptions of personal networks graphs. Following an experimentation approach, we compare the reactions and interpretations of 173 university students when they receive visual feedback of their personal networks with two types of graphics: the visualization of (a) 'strong ties' versus (b) the multiplexity of support providers. We find that viewers generally prefer the social support oriented visualization of their personal networks. The 'multiplexity' graphic allows the detection of key actors, conveys new information, reflects the position of ego in the network and is considered an overall better representation of the personal network. However, the 'strong ties' graphic has a positive effect, allowing the detection of clusters and groups in the personal network. Finally, we discuss the utility of this method for exploring, eliciting and validating the respondent's personal network information.
\end{abstract}

\section{KEY WORDS}

Personal Networks, Graphic Representation, Strong Ties, Multiplexity of Social Support Providers. 